\title{
¿MODERNIZACIÓN INSTITUCIONAL O MOVIMIENTO DEMOCRATIZADOR DE LA EDUCACIÓN SUPERIOR CHILENA? UNA REINTERPRETACIÓN DE LA TRAYECTORIA PREVIA A LA DICTADURA CIVIL-MILITAR (1920-1973)
}

Mauricio Rifo ${ }^{1}$

\section{RESUMEN}

El presente artículo tiene por objetivo reinterpretar las transformaciones en la educación superior chilena entre los años 1920 y 1973 desde una mirada que sostiene que estas transformaciones son un proceso de modernización institucional a la comprensión de este proceso como una lucha del movimiento democratizador de la educación por redefinir cuestiones como la ciencia, la religión, la formación profesional, la investigación, la regulación pública a través del Estado, la vinculación con la sociedad y la valoración de la educación como derecho humano universal desde la acción de estudiantes y académicos/as promotores/as de diseños democráticos para la universidad. La estrategia metodológica empleada en este estudio fue el diseño cualitativo de producción y análisis de datos para analizar e interpretar los datos se

${ }^{1}$ Universidad Tecnológica Metropolitana (Utem), Santiago, Chile. 
utilizaron elementos del análisis semántico y análisis de contenido del discurso.

Palabras clave: historia de la educación, educación superior, movimientos sociales, reforma universitaria, democratización.

\title{
MODERNIZAÇÃO INSTITUCIONAL OU MOVIMENTO DEMOCRÁTICO PARA O ENSINO SUPERIOR CHILENO? UMA REINTERPRETAÇÃO DA TRAJETÓRIA ANTERIOR À DITADURA CIVIL-MILITAR (1920-1973)
}

\section{RESUMO}

O presente artigo tem por objetivo reinterpretar as transformações na educação superior chilena entre os anos 1920 e 1973 desde um olhar que sustenta que estas transformações são um processo de modernização institucional à compreensão deste processo como uma luta do movimento de democratização da educação por redefinir questões como a ciência, a religião, a formação profissional, a investigação, a regulamentação pública através do Estado, a vinculação com a sociedade e a valorização da educação como direito humano universal desde a ação de estudantes e acadêmicos/as promotores/as de desenhos democráticos para a universidade. A estratégia metodológica empregada neste estudo foi o desenho qualitativo de produção e análise de dados para analisar e interpretar os dados foram utilizados elementos da análise semântica e análise de conteúdo do discurso.

Palavras-chave: história da educação, ensino superior, movimentos sociais, reforma universitária, democratização.

\section{INSTITUTIONAL MODERNIZATION OR DEMOCRATIC MOVEMENT FOR CHILEAN HIGHER EDUCATION? A REINTERPRETATION OF THE PREVIOUS TRAJECTORY TO THE CIVIL-MILITARY DICTATORSHIP (1920-1973)}

\begin{abstract}
The objective of this article is to reinterpret the transformations in Chilean higher education between the years 1920 and 1973 from a view that these transformations are a process of institutional modernization to the understanding of this process as a struggle of the democratizing movement of education through redefining issues such as science, religion, professional training, research, public regulation through the State, the link with society and the valuation of education as a universal human right from the actions of students and academics / promoters / of democratic designs for the university. The methodological strategy used in this study was the qualitative design of data production and analysis to analyze and interpret the data, elements of semantic analysis and discourse content analysis were used.
\end{abstract}

Keywords: history of education, higher education, social movements, university reform, democratization. 


\section{MODERNISATION INSTITUTIONNELLE OU \\ MOUVEMENT DÉMOCRATIQUE POUR \\ L'ENSEIGNEMENT SUPÉRIEUR CHILIEN? UNE \\ RÉINTERPRÉTATION DE LA TRAJECTOIRE ANTÉRIEURE \\ DE LA DICTATURE CIVIL ET MILITAIRE (1920-1973)}

\section{RESUME}

L'objectif de cet article est de réinterpréter les transformations de l'enseignement supérieur chilien entre 1920 et 1973, dans une perspective de modernisation institutionnelle, en une lutte du mouvement démocratique pour l'éducation visant à redéfinir des thèmes tels que la science, la religion, la formation professionnelle, etc. la recherche, la régulation publique, le lien avec la société et la valorisation de l'éducation en tant que droit humain universel découlant de l'action des étudiants et des universitaires en tant que promoteurs de projets démocratiques pour l'université. La stratégie méthodologique utilisée dans cette étude était la conception qualitative de l'analyse des sources primaires et secondaires.

Mots-clés: histoire de l'éducation, enseignement supérieur, mouvements sociaux, réforme de l'université, démocratisation. 


\section{INTRODUCCIÓN}

Antoni Doménech (1989), parafraseando al filólogo e historiador de la filosofía Von Wilamowitz, escribió que no hay interpretaciones verdaderas y falsas, sólo las hay posibles o imposibles. En este sentido, la pretensión de este texto es incorporar una nueva interpretación posible de la trayectoria larga de la educación superior chilena durante el siglo XX.

El estudio de las trayectorias largas de la educación superior ha sido abordado, principalmente, como (1) una investigación histórica o (2) como una investigación sociológica.

La investigación histórica se encuentra centrada en la identificación de "modelos" de universidad con el objetivo de generar marcos comparativos con las universidades contemporáneas (PESET REIG, 2015). Y por su parte, la investigación sociológica ha tenido como objetivo la delimitación teórica de la educación superior como "sistema estructuro-funcionalmente integrado" para así determinar sus roles (investigación, docencia o extensión), gobernanza (colegiada o corporativa), composición social (élite, masiva o universal) y dimensiones de acción (bienes públicos o bienes privados) (LABRAÑA, 2016).

En el marco de la discusión sobre los "modelos" de universidades existe una división entre quienes sostienen el origen histórico de estos modelos en las universidades de París o Bolonia y aquellos que extienden la distinción de universidad en pueblos fuera de la Europa continental, como es el caso de la University of Al Karaouine en Fes, Marruecos, fundada en 859 d.C. También, es posible extender esta discusión a las instituciones asiáticas (MOUTSIOS, 2012). Sin embargo, la discusión e interpretación histórica de las universidades como modelos tendría su continuidad en lo que se ha definido como los modelos "modernos" de universidad, el napoleónico y el humboltiano. Ambos productos de profundos proceso de construcción de lo que entendemos hoy como un "Estado-nación".

Desde estos modelos, napoleónico y humboltiano, se busca comparar 
las actuales universidades para establecer una línea histórica de continuidad con aquellos modelos. No obstante, es posible sostener que este ejercicio desdibuja el conocimiento histórico de las universidades y esquematiza un presente institucional en contradicción con el "corazón" de las investigaciones históricas, o sea la trayectoria institucional estudiada deja de ser una constatación "única" y se vuelve un cuerpo organizacional estructurado y posible de identificar fuera de su propia historicidad institucional.

La situación anterior de los "modelos", ha dado pie a la determinación teórica de la educación superior como "sistema". Esta nomenclatura no apunta, solamente, a la delimitación de un cúmulo de funciones y dimensiones de una institución, en este caso educativa, sino a la comprensión teórica de que el diseño de las instituciones responde al desarrollo de un programa de características ontogenéticas (crecimiento por etapas) y filogenéticas (ramificación evolutiva). De ahí que se hable de modernización de los sistemas sobre la base de un peligroso ejercicio de homologación del complejo proceso histórico de la humanidad al desarrollo de un organismo.

Estas visiones también permean la investigación en Chile. En este sentido, los estudios de mayor fuerza explicativa para la trayectoria larga de las Universidades en Chile son los estudios de Serrano (1993), Brunner (1984) y Levy (1986).

Serrano (1993), realiza una investigación respecto a la trayectoria de la Universidad Real de San Felipe en tiempos de la colonia y su continuidad en la Universidad de Chile durante la formación de la república. En esta dirección, de acuerdo con la autora, el devenir de la universidad en la joven República de Chile estuvo marcada, más que por una posición ideológica específica, por su relación con el Estado. De ello concluye que la transformación de la Universidad de Chile desde 1844 a 1879 es un complejo proceso de consolidación desde una universidad académica a una universidad profesionalizante que marcaría buena parte del desarrollo de la universidad durante el siglo XX y sus consiguientes reformas, dando cuenta de una interpretación que rondaría en la concreción de 
modelos de universidad para el caso chileno.

Para Levy (1986) la trayectoria de las universidades en Latinoamérica puede ser analizada desde el rol del sector privado y, al igual que Serrano (1993), las formas en que este ha interactuado con el Estado. De esta forma, el autor sostiene que existirían tres "olas privatizadoras" en el continente con particularidades en los países analizados por él, a saber: Chile, México y Brasil.

La primera ola privatizadora, inaugurada por la fundación de la primera Universidad Católica del continente en el Chile de 1888, es producto de una diferenciación al impulso laico de las universidades públicas. La segunda ola privatizadora, que tendría su mayor desarrollo desde 1930, es la creación de instituciones privadas elitarias-laicas cuyo objetivo es la formación empresarial y la dotación de funcionarios al Estado. Y la tercera ola privatizadora es aquella de crecimiento de universidades privadas masivas que disputan a las universidades públicas el predominio en el sector.

En esta dirección, Levy (1986) concluye que es posible identificar tres patrones básicos de organización de las instituciones públicas y privadas en Educación Superior. De acuerdo con el autor el principal patrón es la diferenciación entre instituciones con la particularidad de una diferenciación que promueve la educación privada como exclusiva y aquella que diferencia la educación privada como inferior a la formación en instituciones públicas. De este marco de diferenciación, se destaca la particular trayectoria de Chile por su homogeneidad entre el sector público y privado. Levy argumenta que esta homogeneidad se produjo por el significativo rol de las universidades católicas como las principales del sector privado y su vínculo con el Estado chileno.

Para Brunner (1984), la cultura nacional se encontraría, al momento de la reforma de 1967, en una pugna por la modernización del país, por la renovación de la cultura católica y presionada por una renovación generacional. Sin embargo, de acuerdo con Brunner (1984), la reforma habría tenido cierta autonomía relativa respecto al escenario general de renovación. En este sentido, estaba determinada, principalmente, por factores internos de modernización 
institucional reflejada en tres posiciones: la academicista, la tecnocrática burocrática y la reformista, resultando esta última la de mayor dinamismo interno, pero también la de mayor carga traumática para la sociedad. Es desde estos factores internos, o dinamismo propio de la educación superior, que Brunner entenderá que el conflicto por la educación sería un dispositivo en permanente reordenamiento para el posicionamiento de una clase social específica, que a su vez daría cuenta de una particular y estable legitimidad social.

Desde estas interpretaciones de trayectoria larga de las universidades en Chile, el modelo de la universidad profesionalizante del siglo XX, la homogeneidad público-privada del sistema de educación superior de Chile y la modernización institucional del sistema, emergen matices y contribuciones.

Las formas explicativas antes descritas deben ser matizadas y llevadas a una reinterpretación debido, principalmente, a una predefinición de modernización institucional que no ha tenido un registro empírico, así como también, una comprensión de los procesos políticos como meros campos de fuerzas con escaso sentido histórico.

Para el caso de la modernización institucional se formuló un análisis que puso su énfasis en la presión productiva del país en el marco de los modelos de desarrollo industrializador. Tal situación histórica, reconocible en el Chile pos 1938, trajo consigo una "extremización" de esta dinámica en donde se entendió, de manera rígida, que el crecimiento industrial traería, de manera inevitable, un crecimiento de la educación superior o una redefinición de su rol. En esta dirección, Schofer \& Meyer (2005) y Wyatt (2005) identifican esta visión dentro de la educación como parte de una tradición funcionalista. Esta tradición, de acuerdo con Schofer \& Meyer (2005) y Wyatt (2005), no ha logrado demostrar efectos significativos de la industrialización o el desarrollo económico nacional en la expansión de la educación en cualquier nivel ni tampoco una relación de expansión entre las estructuras ocupacionales, los requisitos de habilidades laborales o las demandas del mercado laboral que 
crearían la necesidad de una expansión masiva de la educación superior.

Por los motivos antes expuestos, se propone una compresión del proceso de transformación como un movimiento democratizador, en el sentido que propone Silva Pinochet (2017) ${ }^{2}$ y en la especificidad de la educación como sostiene Carlos Huneeus (1988) 3 , o sea como un complejo proceso de lucha por la universalización de la educación y la cultura cuya manifestación ${ }^{4}$, en cuanto

\begin{abstract}
2 “[...] retomar un análisis que re articule el componente normativo sobre democracia, con el análisis histórico y las condiciones materiales que hacen posible evaluar la extensión misma de la democracia" (SILVA PINOCHET, 2017, p. 89).

3 "En segundo lugar, se hizo un enorme esfuerzo por promover la investigación científica y tecnológica que la universidad tradicional no apoyaba adecuadamente, lo cual se tradujo en la destinación de una gran cantidad de recursos para el aumento de las cátedras de jornada completa o de dedicación exclusiva. En tercer lugar, se buscó promover el desarrollo y el intercambio cultural con la sociedad [...] En cuarto lugar, la reforma implicó una importante reorganización administrativa, pues la universidad [...] se organizó en torno a áreas del conocimiento, lo cual colocó a los departamentos como la base fundamental [...]. Por último, la reforma significó una amplia democratización del gobierno universitario, expresada mediante la participación de la comunidad académica [...] en las decisiones de los órganos de poder, compartiendo la responsabilidad en ellas" (HUNEEUS, 1988, p. 10-11).

4 Créamelo, la palabra "masificación" no me gusta; suele tener un sentido peyorativo; se le asocia - indebidamente y por elementos retardatarios - con los conceptos "degradación", "pérdida de calidad", "desaparición de las excelencias" ... Me parece mejor hablar de democratización de la educación y de la cultura. ¿Esto ...? Sí, efectivamente ocurrió o, a lo menos se avanzó vigorosamente en tal sentido. Se rompieron entonces, de manera progresiva y profunda, los esquemas elitistas, aristocratizantes, limitados y clasistas sostenidos secularmente por los elementos más conservadores; éstos hicieron siempre cuanto estuvo de su parte por restringir el acceso popular a la educación. Recuerde, por ejemplo, que obstruyeron durante veinte años el despacho de la ley de instrucción primaria obligatoria y gratuita.
\end{abstract}

Hubo, pues, democratización creciente de la educación nacional, sobre todo en los niveles básico y medio.

Ahora - y aquí hay una cuestión que merece ser estudiada con detenimiento- quienes se incorporaban al sistema y lograban recorrerlo totalmente, no tenía otra meta que la Universidad. Por consiguiente, la aspiración y luego la presión por que ésta se abriera más, multiplicara y diversificara sus servicios, alcanzó una intensidad impresionante. Tanto en la universidad como fuera de ella, esta presión dio origen a forcejeos tensos entre quienes no se arredraban frente a nuevas responsabilidades ni se sentían con autoridad para bloquear un fenómeno que emanaba del fondo mismo de la sociedad, y los que pretendían que la universidad se mantuviera sorda frente a urgentes requerimientos colectivos.

En estas circunstancias, las ocho universidades - dos estatales y seis privadas - debieron abrirse, lo que originó su rápido crecimiento. Ya hacia 1970, su conjunto constituía un sistema nacional compuesto por una cincuentena de sedes y sub-sedes que prácticamente cubrían al país; concibieron y pusieron en marcha múltiples programas de formación profesional. La función universitaria adquirió, entonces gran complejidad e indudable riqueza. Pero se plantearon muchos problemas; estructuras creada en el siglo diecinueve, retocadas y aún ampliadas, se fueron tornando cada día más estrechas, inadecuadas e insuficientes; hubo desajustes de todo tipo que no podían ser corregidos sino mediante cambios muy profundos. Y 
diseño institucional, tiene, necesariamente, la obligación de reformar la institución existente pero en el marco histórico de estas luchas de estudiantes, académicos/as y sociedad civil por una (1) valorización específica de la ciencia, (2) un crecimiento del rol del estado y una (3) promoción virtuosa de la educación para la sociedad. Son estos tres elementos los que cruzan la democratización de la educación superior en Chile y articulan un vínculo entre los movimientos educacionales de principios de siglo con la década del 60 (SCHOFER \& MEYER, 2005, p. 900).

Es por lo anterior que compartimos la visión de Rivera Tobar (2013) respecto a una relación de continuidad entre una generación azotada por los convulsionados eventos de principios del siglo XX junto a una nueva generación crítica de los resultados de esos procesos. En este marco, las autoridades de las universidades, durante las décadas del 60, convivían con visiones problematizadoras de la situación de las universidades, cuya valorización oscilaba entre posiciones de cambio (democráticas y oligárquicas) y/o mantención (conservadoras) del régimen institucional en general. Tal situación, dotó al movimiento estudiantil de un interlocutor institucional para llevar a cabo su crítica y la concretización de las reformas. De esta forma, se fraguó un consenso parcial de un déficit democrático, y consiguiente amplitud, en las instituciones de Educación Superior chilenas (HUNEEUS, 1988).

En definitiva, la distinción entre reformas modernizadoras y movimiento democratizador es sustantiva para poner en cuestión una interpretación de la trayectoria de las universidades como mera "modernización institucional" que establece puntos de encuentro, entre otros, como proyectos de financiamiento o convenios de cooperación entre las universidades5. Tales eventos no responden, necesariamente, al sentido del movimiento democratizador y revelan la estrechez analítica de la compresión del

aquí encontramos una de las raíces de la reforma iniciada en 1967. Entrevista Hernán Ramírez Necochea. Revista Araucaria de Chile, n. 3, 1978, p. 100.

5 Para esta visión, el punto de relación con los eventos de 1967 tiene antecedentes frustrados, por la imposibilidad de resolver el caos institucional, con los convenios que realizó la Universidad de Concepción (privada) en 1959 con la Unesco y la Fundación Ford. 
movimiento de cambio universitario como un problema de modernización institucional, entendido, en clave política, como reforma.

\section{LA LUCHA ESTUDIANTIL Y ACADÉMICA POR DEMOCRATIZADOR LA EDUCACIÓN SUPERIOR CHILENA DURANTE EL SIGLO XX (1906-1973)}

Las universidades chilenas entre 1967 y 1973 se encuentran atravesadas por una serie de fenómenos sociales y políticos de cambio. A su vez, las mismas casas de estudios han sido el espacio para la trayectoria de una serie de militantes políticos de diversas agrupaciones y partidos del país. Es por este motivo, que la universidad tensada desde la contingencia histórica y dinamizada por los diversos militantes en diversas áreas de la institución se vuelve un escenario de la lucha por democratizar o conservar su funcionamiento y sus fines.

El principal enfoque para abordar la cuestión de las expresiones políticas y su politización en la Universidad es aquella que asocia este proceso a la conformación de nuevas élites (VALENZUELA VAN TREEK, 2011; GAZMURI, 2001; HUNEEUS, 1973). Estas nuevas élites serían producto de la condición juvenil de los estudiantes junto al flujo de ideas teóricas que recorre a las universidades.

A su vez, la forma de organización política dentro de la universidad se ha observado desde tipos de representación estamental (Cifuentes, 2004), principalmente estudiantil, y los orígenes y trayectorias de diversas fuerzas sociales que devienen en partidos políticos o tendencias influyentes en la sociedad en diversos niveles (VALDIVIA DE ZARATE, 2008; CORREA, 2008; MOYANO, 2009).

Ante lo anterior, se opta por la comprensión de estos grupos universitarios como intelectuales en el sentido de Antonio Gramsci, o sea con 
capacidades técnicas y de dirigencia en la sociedad, más que como representantes o miembros de la una élite "auto reproducida" (GRAMSCI, 1967, p. 21-32). Tal conceptualización permite comprender al movimiento de estudiantes bajo un marco político plural que reconoce posiciones y funciones de conservación o transformación.

Para el caso del mundo académico aún resulta complejo poder abordarlo a través de fuentes secundarias (AUSTIN, 2004; SUBERCASEUX, 2008; CRISTI \& RUIZ, 1992; MORRIS, 1966). Esto se debe principalmente a que la reconstrucción histórica del mundo intelectual chileno ha estado asociado a la población migrante, muy influyente cabe recalcar, y a los circuitos intelectuales partidarios cuya producción si bien comparte nicho en la universidad no están apuntados, estrictamente, a la misma (ANGELL, 1972). Tal vez sea posible sostener que es más bien una tendencia general en el mundo intelectual nacional, de la primera mitad del siglo XX nacional, de tener una mirada centrada desde la sociedad hacia los diversos espacios o instituciones, en este caso de la educación superior. Sumado a esto, resulta aún poco desarrollado el impacto disciplinar y los marcos de politización que esto puede traer, como es el caso de la migración de intelectuales brasileños durante la década del 60 chileno que traían consigo la teoría de la dependencia o la pedagogía crítica.

Por consiguiente, se considerará el rol del movimiento estudiantil como central para entender la democratización de la universidad en cuyo espacio de crecimiento se encuentran los académicos que participaron en contra o a favor, como estudiantes y después de serlo, de los procesos políticos universitarios chilenos. Sin embargo, cabe recalcar que aquello es parte de la trayectoria de las universidades chilenas, pero no es posible extenderlo como un conjunto de axiomas teóricos posibles de hacer generalizable a todas las trayectorias de las universidades.

En la dirección anterior, existe un consenso en entender al movimiento estudiantil chileno como el principal actor político de la universidad bajo una trayectoria que tiene uno de sus eventos fundantes en la conformación de la 
Federación de Estudiantes de Chile (FECH), durante una protesta callejera de 1906, y un ethos transformador, como diría Enrique Molina, de un "movimiento intelectual de carácter social, científico y filosófico" (BIAGINI, 2002, p. 282). Esta situación daría cuenta de un actor político histórico que escapa a la sola condensación del "momento democratizador" de 1968 (CIFUENTES, 2004).

De esta forma, desde sus inicios la politización del movimiento estudiantil ha respondido a una pluralidad de expresiones:

[...] Entre los universitarios había radicales, masones, anarquistas, vegetarianos, liberales algunos socialistas, colectivistas, nitzcheanos, estirnianos, espiritistas, católicos, nacionalistas, arbitristas y muchachos casi silvestres [...] (GONZÁLEZ VERA, 1945, p. 2).

Sus lazos con la sociedad civil, en especial con los sectores populares, se vislumbra desde los años fundantes de la FECH. Ejemplo de aquello es la creación, en 1910, de la Universidad Popular Lastarria (UPL) que tenía como objetivo la "educación mutua y libre" entre obreros, artesanos y estudiantes.

Este tipo de vinculación se extendió incluso a la unidad de reivindicaciones junto a la Federación de Obreros de Chile (FOCH) durante la conformación de una Asamblea Obrera de Alimentación Nacional (AOAN) producto del monopolio de comercio (1920) y la participación en la conformación de una asamblea constituyente auto convocada por las fuerzas de la sociedad civil durante la crisis de régimen en 1925 (BASTÍAS, 2007, p. 21).

En este marco, la compresión del movimiento estudiantil chileno ha sido analizado desde polos aparentemente opuestos: (1) la compresión de "un" movimiento estudiantil o de "varios" movimientos estudiantiles y (2) la conformación del movimiento estudiantil como producto de la "cuestión social" o con autonomía relativa al contexto social (GÁRCES, 2012; GARRETÓN \& MARTÍNEZ, 1985; CÁRDENAS TOMAZIC \& NAVARRO OYARZÚN, 2013; CIFUENTES, 2004; BIAGINI, 2002). 
Para efectos de este escrito comprenderemos la existencia de "un" movimiento estudiantil, históricamente registrable, con una autonomía relativa al contexto social en el marco de sus diversos procesos de democratización de la Educación Superior. En este sentido, de acuerdo con Cifuentes (2004), el movimiento estudiantil chileno debe ser visto como una trayectoria de democratización de la educación superior desde 1920, sustentada por diversos procesos de reforma enmarcados en discusiones de facultades, departamentos o institutos o de las casas de estudios en general hasta la radicalización o decantación del proceso en los años 1967 a 1968.

En el marco anterior, el movimiento estudiantil chileno incubó una serie de posiciones políticas que fueron dotando de mayor coherencia los propósitos de los diversos estudiantes. Cabe destacar que la mayor o menor presencia de posiciones políticas más o menos relevantes debe entenderse en un plano general de pluralidad política institucional.

Los primeros años del movimiento estudiantil chileno se encuentran marcados por una influyente presencia de posiciones políticas ácratas, anarquistas y libertarias. La generación universitaria de los años veinte nacionales es ampliamente reconocida por su vinculación a un ambiente cultural de vanguardia junto a un compromiso social con las clases más pobres de la población y el movimiento obrero nacional (BASTÍAS, 2007). Es en este contexto, que los/as estudiantes ácratas, anarquistas y libertarios construyen una identidad política a la primera federación de estudiantes de chile y la vinculan al acontecer nacional, pero al mismo tiempo promueven una democratización de la educación superior en sus propias casas de estudios (CIFUENTES, 2004).

La pluralidad política de la universidad no estuvo exenta de enfrentamientos violentos. Con la llegado de 1920 se desata una coyuntura electoral y política general de gran relevancia para la universidad. El proceso electoral presidencial de 1920 trae consigo una maniobra política del Ministro de Guerra de la época, Ladislao Errázuriz, quien, ante el inminente triunfo del 
candidato opositor, Arturo Alessandri, realizó un movimiento de tropas ante la amenaza de un supuesto espionaje peruano. Esta situación ha sido históricamente verificada como falsa (SENADO UNIVERSITARIO, 2012).

No obstante, la situación golpea a la federación de estudiantes debido a su posición pública de rechazo ante la denuncia del gobierno. Por este motivo, los grupos nacionalistas, católicos conservadores y parte de la oficialidad del ejército nacional arremeten contra la sede de la federación, el 21 de Julio de 1920, saqueándola y quemándola con el respaldo presidencial. Tal situación, se prolonga durante la noche del mismo día y provoca la muerte del estudiante conservador de la Universidad Católica de Chile, Julio Covarrubias. Este evento provoca la reacción desmedida y contra derecho de las autoridades de la época y ordenan el arresto de los estudiantes y obreros cercanos a la Federación de Estudiantes de Chile (FECH).

[El ministro en visita José Artorquiza y Líbano] condujo en pocos días a un centenar de obreros y estudiantes a poblar las prisiones inmundas de la capital; y sus rigores deliberados llevaron al hospital a Pedro Gandulfo, a la Casa de Observación de la Casa de Orates a Evaristo Lagos, y al Cementerio, de donde no se vuelve, a José Domingo Gómez Rojas [...] Los frutos emponzoñados siguen produciéndose. En el cerebro de Isidro Vidal ha florecido la locura, como la necesaria consecuencia de once meses de cárcel y de vejaciones (SCHWEITZER, 1921, p. 4).

Dentro de los detenidos se encontraba el influyente joven poeta José Domingo Gómez Rojas, quien fue torturado y aislado en prisión, provocándole un deterioro mental que lo llevó a la locura y la muerte en septiembre del mismo año (1920). La trágica muerte movilizó al movimiento democrático por la educación y decantó en un proceso de crítica al gobierno de Chile y a la situación de la universidad en general. Cabe mencionar que este evento marcó a la generación más premiada en el contexto literario que ha tenido chile en figuras como Neftali Reyes (Pablo Neruda) o Manuel Rojas, debido a su relación de amistad estrecha con José Domingo Gómez Rojas. 
La democratización de la educación superior, promovida por los/as estudiantes, no responde a un acontecer meramente contemplativo o retórico, sino más bien como respuesta a la constante restricción asociativa a los diversos estamentos no directivos de la universidad.

Es decir, el Consejo de Instrucción, ante las deficiencias materiales y el
crecido número de universitarios, no mejora aquellas, pues sin duda
no le conceden dinero, iya que la defensa del país movilizó millones! ...
Y resuelve llanamente: ino más alumnos! Una Universidad que
retrocede en el momento mismo en que la intelectualidad entera se
hace más atrevida [...] (ANÓNIMO, 1920, p. 10).

Es este tipo de situación la que gatilla, el 20 de junio de 1922, una declaración del pleno de estudiantes de la Universidad de Chile en contra de la resolución que adoptó el Consejo de Instrucción Pública de prohibir la reunión de las escuelas universitarias salvo bajo la aprobación de determinadas materias. La declaración de los/as estudiantes postulaba:

1. Autonomía de la Universidad. La organización y dirección de la Universidad debe generarse exclusivamente en los profesores, alumnos y egresados.

2. Reforma del sistema docente. Se postula la necesidad de la docencia y la asistencia libres.

3. Revisión de los métodos y del contenido de los estudios. Cada centro estudiantil debe nombrar comisiones especiales para que en cada facultad se estudie esta revisi6n.

4. Extensión Universitaria. La Universidad debe vincularse a la vida social difundiendo las ciencias, la filosofía y las artes (BARRERA, 1968, p. 628).

Los y las estudiantes de la Universidad de Chile continuaron su manifestación con semanas de paralización, le llamaron la "semana universitaria", y procesos de fortalecimiento interno que trajo consigo la suspensión y expulsión de estudiantes: 
En vista de los actos de incitación y de violencia cometidos durante los días de desórdenes estudiantiles, el Consejo acuerda aplicar los siguientes castigos disciplinarios: Expulsión de todas las escuelas universitarias y suspensión del derecho de rendir exámenes válidos hasta marzo inclusive de 1924, a los siguientes estudiantes: Eugenio González, Enrique Matta Figueroa, Julio Barrenechea, Oscar Schnake, Oscar Acevedo y Alfredo Larraín Neil. Prohibición de asistir a clases a ningún establecimiento universitario y suspensión de exámenes hasta diciembre inclusive de 1923 a los estudiantes: Isaac Etchegaray y Moisés Cáceres. Igual medida a la anterior se acuerda tomar contra los alumnos que promovieron desórdenes y patrocinaron una huelga en el liceo de hombres, de San Bernardo. Ellos son: Oscar Cárcamo, Jorge Videla, Fernando Meyer, Ricardo Videla y Eduardo Videla (ANÓNIMO, 1922, p. 3).

\section{Esta situación dilata el proceso de democratización y lo reorienta en} defensa de los/as estudiantes sancionados, sin embargo, y lejos de los objetivos de los y las estudiantes envueltos en el proceso, se reconocen algunos cambios inorgánicos en partes de la comunidad universitaria:

Pero entretanto, vale la pena anotar que la preocupación por la reforma se abre paso, y muchos profesores, -además de la prensa, de algunas Facultades, y del Consejo de Instrucción que estimó conveniente encomendar a los Decanos la difícil tarea de demostrar públicamente las bondades del sistema en actual vigencia- empiezan a revelar una intención reformista, naturalmente incoherente $\mathrm{y}$ descaminada. En efecto: sabemos que el profesor Sierra ha estado estudiando con sus alumnos la manera de introducir algunos cambios en la manera actual de proporcionar la enseñanza. Por su lado, el Doctor Amunátegui, Decano de la Facultad de Medicina, ha suprimido momentáneamente las listas de asistencia, y está sondeando la opinión de sus alumnos para adoptar una actitud definitiva. Y finalmente, el cuerpo de profesores del Instituto Pedagógico ha encomendado a una comisión de su seno, integrada por representantes de los estudiantes, el estudio de una reforma de la enseñanza universitaria, en el sector que le corresponde a dicha Escuela. Evidentemente toda esta actividad es un progreso (SCHWEITZER, 1922, p. 3).

Bajo la complejidad del momento político nacional y la dificultad de sortear los obstáculos institucionales, el movimiento democratizador se diluye 
hasta un nuevo impulso en 1931 y 1932 con motivo de un nuevo escenario de reforma.

La total autonomía en su triple fase: administrativa, didáctica y económica; la docencia y asistencia libre; la extensión universitaria: la participación de los estudiantes en los Consejos y Facultades; la democratización de la Universidad, son puntos que habremos de conseguir como base mínima del nuevo Estatuto. Representamos una parte del electorado estudiantil, consciente y decidido; iremos a la Comisión con firmeza y energía y los universitarios, esta vez, no se dejarán engañar ni arrastrar por autoridad ni promesa alguna (VARIOS, 1931, p. 2).

Se suma a ello eventos posteriores de huelgas y conflictos estudiantiles, en 1944, como el vivido por los y las estudiantes de arquitectura que promovían un cambio en los contenidos de enseñanza en la arquitectura extendiéndose hasta estudiantes del Instituto Pedagógico de la Universidad de Chile (BARRERA, 1968, p. 632). Algunas de las consecuencias del movimiento del Instituto Pedagógico fueron:

Se fusionan el Instituto Superior de Humanidades y el Instituto Pedagógico en un Instituto Pedagógico, concentrándose en este los ramos especiales y los generales. En vez de un plan de estudios de cinco años, se organiza uno de cuatro, reservándose el quinto para la práctica profesional intensiva. Se concentran varias micro-cátedras en cátedras uniformes de 6 y 3 horas. Se organiza un plan de estudios que provee una formación paralela en la especialidad y en lo pedagógico. Con anterioridad, los tres primeros años correspondían exclusivamente a la especialidad y los dos últimos a lo pedagógico, existiendo entre ambos periodos una neta desvinculación. Se crean preseminarios y seminarios adscritos a las cátedras. Se crean cátedras paralelas en todas las materias generales y en algunas de la especialidad. Ingresan varios profesores nacionales y extranjeros de prestigio. Para obtener el título se exige solo una memoria en vez de dos. Se crea la Comisión de Docencia, con representación estudiantil. También se aprueba una mayor representación estudiantil en la Facultad. Se consiguen fondos para la adquisición de un nuevo local (BARRERA, 1968, p. 631-632). 
En general, el movimiento democratizador, anterior a 1968, pujó por dar contenido disciplinar y social a sus diversas especialidades de conocimiento junto al compromiso social con el país promoviendo una democratización en la formación disciplinar y en la universalización de la cultura y el conocimiento. En este sentido, la afirmación de un movimiento democratizador en contacto con generaciones críticas devenidas en docentes y autoridades universitarias es perfectamente reconocible en figuras como Eugenio González, uno de los estudiantes sancionados en 1920 que para los años sesenta fuera rector de la Universidad de Chile. Sin embargo, este punto de diálogo generacional no estuvo exento de dificultades que dieron origen a una serie de quiebres en las diversas tradiciones políticas del país y de la propia universidad.

La destacada generación del 20 fue hegemónicamente anarquista y socialista, la generación del 60 fue, predominantemente, marxista en sus diversas "corrientes".

Las diversas corrientes de pensamiento del país como la socialcristiana (Democracia Cristiana), socialista (Partido Socialista de Chile), comunista (Partido Comunista de Chile), libertaria, hispánico-católica (Partido Conservador), radical (Partido Radical) y liberal (Partido Liberal) fueron fuertemente sacudidas por sus juventudes (VALDIVIA DE ZARATE, 2008).

De estos movimientos, fuertemente vinculados a las universidades, nacen una serie de nuevas expresiones políticas como el movimiento de izquierda revolucionaria (MIR) cuyo núcleo se encuentra en la Universidad de Concepción y gran parte de sus miembros fue parte de las juventudes del Partido Socialista o disputaba con la cultura comunista. El Movimiento Acción Popular Unitaria (MAPU) de la Pontificia Universidad Católica y de origen demócrata cristiano o el movimiento de Acción Gremial también de la Pontificia Universidad Católica y parte de la crisis de los partidos tradicionales de la derecha chilena (MOYANO, 2009; VALDIVIA DE ZARATE, 2008).

Por parte del MIR, su fuerte vínculo a las tradiciones socialistas, trotskistas y libertarias de la política chilena lo encausó como un movimiento 
político de corta duración (1965-1974), pero de particular relevancia por su posición de crítica ante el proceso chileno encabezado por los partidos de izquierda presentes en la alianza electoral que llevará a Salvador Allende a ser presidente de Chile, la Unidad Popular (UP).

Uno de los principales componentes que vinculan al MIR al movimiento estudiantil es que buena parte de su dirección política más representativa, consolidada en 1968, pertenece directamente a la Universidad de Concepción en figuras como Miguel Enríquez, Bautista Van Schouwen, Nelson Gutiérrez o Luciano Cruz.

Por el motivo anterior, el principal bastión político estudiantil del MIR fue la Universidad de Concepción a través de la presidencia en la federación de estudiantes de Luciano Cruz (1967) y Nelson Gutiérrez (1968). Para el caso de la Universidad de Chile su presencia fue menor, siendo su mejor resultado el de 1.300 votos en las elecciones FECH de 1968, por detrás del Partido Comunista (3.177) y el Partido Socialista (1.687), consolidándose como la tercera fuerza de izquierda en la Universidad de Chile.

El eje discursivo y político del MIR, para la educación superior, fue la construcción de una Universidad Militante que se entroncará con el proceso político general de la sociedad chilena que Nelson Gutiérrez denominó "la segunda etapa de la reforma universitaria" (NELSON GUTIÉRREZ, 1971, p. 4). Este proceso consistía en un diagnóstico de una educación superior fuertemente concretada en la formación de las élites y clausurada para los sectores populares nacionales. Tal situación debía ser revertida a través de un cambio de eje en el foco de la educación superior para contribuir, desde la función de la universidad, a la liberación de obreros y campesinos nacionales desde procesos de educación científica universales.

Por su parte el Mapu, como sostiene Moyano (2009), su también breve pero influyente trayectoria se encuentra marcada por una ruptura generacional y de dirección de una tradición socialcristiana plasmada en el partido demócrata cristiano (DC). Este cambio se produce, principalmente, por una crítica al 
gobierno de Eduardo Frei en la ambigüedad y giros del presidente. Tal situación

llevará a la Democracia Cristiana a una fuerte discusión ideológica que traerá consigo su quiebre en 1969 de parte de la dirección fundadora del partido y la mayoría de la juventud del partido. Tal evento dará fundación al Mapu6 ${ }^{6}$.

En el plano educacional, el Mapu promovió la masificación de la Universidad y buscó dotar de contenido a su función desde una visión de Universidad con compromiso social (VALENZUELA, 2011). Ambas aristas estuvieron relacionadas con el rol decisivo que jugaron los miembros del Mapu en la administración de la Unidad Popular (UP) y en las direcciones de extensión e investigación de la Universidad Católica de Chile junto a una tradición socialcristiana de compromiso con los más pobres de la sociedad7.

En una vereda política contraria, pero en la misma Pontificia Universidad Católica de Chile, el movimiento gremial se funda como una acción política anti democratización de la universidad y, al mismo tiempo, de refundación de la derecha chilena (VALDIVIA DE ZARATE, 2008).

Su principal organizador es él joven estudiante de leyes, Jaime Guzmán. La trayectoria de Guzmán es central para entender la articulación del

\footnotetext{
6 "Los historiadores lo ubican como el grupo rebelde que se escindió de la DC que incluyó a los jóvenes rebeldes marxistizados de la JDC -Rodrigo Ambrosio estudió con Althusser y ya planificaba la revolución socialista aun en la dc (J. Gazmuri, 2000) -, pero también a los parlamentarios Rafael Agustín Gumucio, Julio Silva Solar y Alberto Jerez. La historiadora Cristina Moyano documenta el protagonismo de la generación joven (los parlamentarios se mudan a la izquierda cristiana, huyendo de la dogmatización): la generación entusiasmada con las revueltas de los años sesenta, fascinados por el poder y la juventud que quiere reformar las universidades, el agro y luego hacer la revolución (Moyano, 2009). el historiador Cristián Gazmuri (2003) ve al MAPU como un subproducto de la universidad católica de santiago, como lo fue también la udi en sus vertientes de gremialistas y Chicago boys. Huneeus (1973) demostró el vínculo directo entre el movimiento estudiantil y los grupos políticos en chile. Irene Agurto comparte que el movimiento estudiantil de la UC fue la gran escuela formadora del estilo y la "génesis cultural de la élite mapucista (1991: 118-119)" (VALENZUELA VAN TREEK, 2011, p. 190).

7 "Hacia fines de 1970 asume como nuevo presidente de la Federación de Estudiantes de la Universidad Católica de Valparaíso Gonzalo Pineda, militante del MAPU, al igual que fue electo presidente de la Federación de Estudiantes de la Universidad Técnica Federico Santa María Etienne Lefranc, en mayo de 1971. Los triunfos estudiantiles, que fueron los más significativos de la colectividad, nos demuestran el importante influjo que el MAPU generó en los sectores juveniles universitarios, donde tuvo sus principales bases de apoyo, aun cuando se declarara como partido proletário" (MOYANO, 2009, p. 285).
} 
movimiento gremial de la Pontificia Universidad Católica como una "cantera" (MUÑOZ TAMAYO, 2014, p. 199-205) refundacional de la derecha chilena ante el retroceso de posiciones jerárquicas de la iglesia católica y el estancamiento político-económico de la derecha nacional.

Guzmán articuló un movimiento político que buscó re-jerarquizar la vida civil en estamentos cuasi-feudales, por ese era contrario al cogobierno universitario, y un orden constitucional que blindará la propiedad privada, de ahí su rechazo férreo a cualquier tipo de democratización. En sus palabras:

La institución universitaria tiene una naturaleza propia y muy definida, por muchas que sean las diversas formas que haya adoptado en el tiempo y en el espacio. En ella hay un núcleo insustituible: la comunidad de maestros y discípulos (ROJAS, 2012, p. 19).

Su trayectoria y la del movimiento será central para entender el devenir de la dictadura civil-militar y en especial en la orientación de la síntesis neoliberal e hispánico-católica en el sistema de ideas de la reforma a la educación superior de 1981.

En definitiva, el movimiento estudiantil ha actuado, a lo largo de la historia de chile, como un cuerpo intelectual plural cuya acción es gravitatoria en la dirección que tome el país junto a la construcción, implícita, de un puente intergeneracional que bajo ciertas circunstancias y con relativa autonomía harán síntesis en movimientos educativos democratizadores 0 antidemocratizadores. 


\section{LOS FUNDAMENTOS DEL MOVIMIENTO DEMOCRATIZADOR UNIVERSITARIO DE 1967-68: CIENCIA, PROFESIONALIZACIÓN, DESARROLLO Y TERCERA MISIÓN}

El principal hito del movimiento democratizador en la Educación Superior chilena se concentra en la ocupación de la casa central de la Pontificia Universidad Católica de Chile, en el año 1967. La ocupación tuvo como motivo la imposibilidad de diálogo entre un grupo de estudiantes de la universidad con las autoridades. Lo más relevante del acto es la condición misma de la universidad interpelada, ya que respondía (y aún responde) a una propiedad eclesiástica conformada en oposición institucional y normativa a la universidad pública del país, la Universidad de Chile. Tal condición la situaba en un perfil conservador y de formación específica de élites con bajo compromiso democrático y público.

Es en este contexto institucional, influenciados por el Concilio Vaticano II y las Encíclicas de Juan XXIII y de Pablo VI, pero, principalmente, por el escrito conciliar elaborado en la ciudad de Buga, Colombia, titulado Gadium Spe, que promueve una visión científica de la teología y una misión de compromiso social, desde la cultura y la crítica, con los pobres (RIVERA TOBAR, 2013, p. 9). Los y las estudiantes de la Pontificia Universidad Católica debaten y se suman a la necesidad de democratizar el restrictivo horizonte de una universidad para una élite conservadora.

En este sentido, el movimiento democratizador de la Pontificia Universidad Católica responde a un desplazamiento conciliar de la Iglesia Católica junto a la necesidad de dotarse de instituciones, representantes y procesos de conocimiento científico-teológico que la universidad no promovía. Ejemplo de aquello, es lo llevado a cabo el 15 de junio de 1967 en la Universidad Católica de Valparaíso donde se crea el Instituto de Teología que tuvo como objetivo dar espacio a la mirada desde la religión en el quehacer científico e intelectual de la universidad (ALLARD, 1997, p. 13). Sumando al evento más épico del proceso con la ocupación de la casa central de la Pontificia 
Universidad Católica de Chile el 10 de agosto de 1967. Tal evento, provocado por la ausencia de respuesta a la demanda de un Vicerrector laico en la Universidad, trajo consigo la reformulación jerárquica de la misma y consagró la elección del rector, Fernando Castillo Velasco, quien señalaba que en su administración:

Se acentuará la importancia de la Ciencia, la democratización en el mando y el acceso a las aulas; especial realce se concede a la función crítica frente a las alienaciones que asaltan al hombre y a los grupos sociales, y mayor acento aún se dispensa al papel de inserción de la universidad en los procesos que suceden en su exterior (UNIVERSIDAD CATÓLICA DE CHILE, 1968, p. 7).

La democratización de la Pontificia Universidad Católica tuvo como eje central la redefinición de la misión católica en un sincretismo con el conocimiento científico y teológico de la búsqueda de la verdad. Tal horizonte tuvo como efectos concretos (1) un grupo de estudiantes comprometidos con los pobres $^{8}$, (2) cambios curriculares e institucionales de la organización del conocimiento en su dimensión investigativa ${ }^{9}$ y (3) la apertura de las jerarquías concentradoras en la toma de decisiones de la institución.

Durante el mismo año (1967) de los eventos que sacudían a la Pontificia Universidad Católica, la Universidad Técnica del Estado vivía un proceso de democratización bajo otro eje, pero en sintonía con la incorporación institucional de nuevas dimensiones de la universidad y mecanismos de

\footnotetext{
8 "En 1968 Universidad Católica creó con el apoyo e iniciativa de estudiantes y egresados de la Escuela de Educación, y con el apoyo del rector Castillo Velasco se creó el Departamento Obrero y Campesino (Duoc), a fin de "proveer de oportunidades a todos los chilenos en cuanto al ingreso al sistema educacional". El Duoc fue concebido como un "puente entre la Universidad Católica y el mundo del trabajo"101, al mismo tiempo que como "base de sustentación social de la universidad y de la proyección de ésta en la sociedad". El Duoc mediante la labor de la Unidad de Instrucción Comunitaria (UNIC) - desarrolló cursos en las poblaciones a través de convenios con organizaciones de base, tales como Centros de Madres, juveniles, sindicatos y asociaciones gremiales, poniendo especial énfasis en las mujeres y dirigentes poblacionales, que también constituyeron una plataforma política para el proyecto de 'Revolución en Libertad’ de Frei” (RIVERA TOBAR, 2013, p. 33).

9 La Reforma en su implementación práctica, logra llevar a efecto la articulación e institucionalización de la Carrera Académica, dictándose el Estatuto del Personal Académico en 1971, aumentándose la planta de profesores con jornada completa (ALLARD, 1997, p. 113).
} 
participación democrática.

Los eventos de la Universidad Técnica del Estado se encuentran en, por una parte, la dilatación en generar cambios institucionales demandados por parte del cuerpo de profesores y la federación de estudiantes de la universidad, y por otra, la oposición a la reelección del Rector, Horacio Aravena.

\begin{abstract}
La FEUT está convencida de que la Universidad Técnica del Estado está suficientemente desarrollada como para generar democráticamente sus autoridades. No podemos estar de acuerdo con que un Consejo Universitario de 23 personas, doce de las cuales pertenecen a entidades extrauniversitarias y de los once restantes, siete han sido nombradas a propuesta del propio Consejo le imponga a la Universidad Técnica del Estado su máxima autoridad por cuatro años.

Por este motivo hemos dicho que no aceptamos un nuevo Rector por un período de cuatro años conforme al actual sistema. La FEUT ha planteado la necesidad de designar un Rector transitorio encargado fundamentalmente de activar y obtener la tramitación externa de la nueva Ley Orgánica y una vez que ésta esté en vigencia, convoque a un Claustro Pleno encargado de elegir al nuevo Rector por un período ordinario $^{10}$.
\end{abstract}

Esta oposición a la elección del rector gatilló la intervención, el 27 octubre de 1967, del gobierno de la época con la creación de una comisión de reforma de la universidad. De esta forma, para 1968 se realizaron por primera vez elecciones de rector. Estas elecciones fueron ganadas de manera sistemática por el ingeniero Enrique Kirberg Baltianky hasta el golpe de estado de 1973 (HUNEEUS, 1988).

La particularidad del movimiento democrático de la Universidad Técnica del Estado, a diferencia de la Pontificia Universidad Católica, se enmarca en la redefinición de la formación profesional de la universidad. Esta

10 Carta abierta de la Federación de Estudiantes de la Universidad Técnica del Estado a los profesores, 25 de septiembre de 1967. Obtenido en Archivo patrimonial Universidad Santiago de Chile (20/05/2017): http://archivopatrimonial.usach.cl/reforma/wp-content/uploads/ 2016/o6/Carta-abierta-de-la-Federaci\%C3\%B3n-de-Estudiantes-de-la-Universidad-T\%C3\% A9cnica-del-Estado-a-los-profesores-25-de-seotiembre-de-1967-Transcripci\%C3\%B3n.pdf. 
reinterpretación es significativa debido, principalmente, a la crítica que el movimiento estudiantil llevaba sistemáticamente desarrollando a una "sobre profesionalización" de la formación universitaria en detrimento de la investigación científica y tecnológica. De esta crítica, la Universidad Técnica del Estado se hace cargo como proceso democrático.

\begin{abstract}
Hemos sostenido, y lo reiteramos, que nuestro objetivo esencial es crea una Universidad Nueva, que no sea una mera fábrica de profesionales, sino que, ligada estrechamente a los grandes avances científico-técnicos de la época, se transforme permanentemente y cumpla una función decisiva de contribución a los cambios sociales y el desarrollo que el país necesita ${ }^{11}$.
\end{abstract}

Desde este eje es posible desprender aspectos de resignificación sobre lo profesional. Un primer aspecto responde a la valorización de la educación como un fenómeno virtuoso para la sociedad y en especial para los pobres por su integración desigual al mundo del trabajo y la cultura.

La participación de los trabajadores en la economía y en los planos diversos del desarrollo social, se haría real y efectiva. Por lo tanto, ese hecho originaba un serio desafío: los trabajadores debían equiparse para participar en forma eficaz y consciente en estas nuevas responsabilidades. La universidad estaba ayudando a la capacitación y formación de estos nuevos cuadros técnicos y políticos que el desarrollo del país iba a necesitar (KIRBERG, 1981, p. 149).

Y un segundo aspecto, es la incrustación de la ciencia y la técnica en lo profesional como el corazón de la misión formativa de la universidad. De esta forma, se rescata lo profesional de una formación poco nutritiva y se vuelve a "llenar" de los principales elementos de avanzada científica y técnica de la época con un sentido de servicio social amplio.

\footnotetext{
${ }^{11}$ Discurso del Rector Kirberg sobre la marcha de la Reforma y la Corporación, 1969. Obtenido en Archivo Patrimonial Universidad Santiago de Chile (20/05/2017): http://archivopatrimonial.usach.cl/reforma/?p=326\#more-326.
} 


\begin{abstract}
Que nuestras manos estudiantiles estrechen las callosas manos de los campesinos, que nuestro sentir se refleje en el espejo cristalino de los ojos infantiles, que nuestra piel conozca del aire, el mar, el sol de nuestros campos, que nuestras venas estén plenas de la satisfacción con el deber cumplido, que nuestro corazón y el del pueblo palpiten al unísono (ANÓNIMO, 1971, p.2).
\end{abstract}

Para el caso de la Universidad de Chile, la Universidad pública por excelencia, el principal eje que articula el proceso de democratización es la función formativa del Estado desde eventos gatilladores similares a la Pontificia Universidad Católica y la Universidad Técnica del Estado.

En mayo de 1968 los estudiantes de la Universidad de Chile, representados por su federación, se opusieron a través de movilizaciones a las decisiones del Consejo Superior Universitario. Tal evento se transformó en el punto de quiebre de una trayectoria de discusión de más larga data respecto al rol de la Universidad Estatal pero que se vive, por la comunidad, en espacios de menor densidad participativa (CASALI, 2015).

Para el caso de la Universidad de Chile existe una visión bastante extendida de que su proceso de reforma responde a un crecimiento institucional (modernizaciones) que tuvo como consecuencia un proceso político de reforma de mayor data junto a un diálogo entre académicos y estudiantes de mayor fluidez, a pesar de los conflictos (CASALI, 2015, p. 69). Si bien es registrable el crecimiento de la institución desde el rectorado de Juan Gómez Millas (19531964), la interpretación de este crecimiento como mero perfeccionamiento institucional no permite comprender el reposicionamiento del rol del Estado en el país, y en el mundo, que se vive desde inicios del siglo XX (DOMÈNECH, 2004). Debido a esto, y para el caso de la Educación Superior, el crecimiento es una expansión de la función formativa del Estado a través de sus instituciones educativas presionadas por diversos cambios sociales.

La decantación de este complejo proceso histórico trajo consigo que el debate de la educación pública universitaria fuera, lentamente, considerando su vinculación con la sociedad como un tema de relevancia central. De aquel 
debate se desprendieron dos aspectos principales: (1) educación superior y desarrollo y (2) educación superior y extensión.

En este sentido, el proceso de democratización de la Universidad de Chile tuvo como síntesis la comprensión de la situación económica del país como marcada por una dependencia estructural cuya consecuencia anexa trajo consigo un atraso cultural a la nación. Es en ese marco, que el debate democrático de la Universidad de Chile levanta sus principales puntos de crítica sobre cómo las instituciones educativas del Estado pueden revertir tal situación.

Pero el sistema económico de una sociedad contemporánea en transformación, en la docente, requería de la universidad la formación de profesionales de diversos niveles y especializaciones. La universidad no los aportó en la cantidad y calidad suficiente, lo cual es una de las causas de que nuestro desarrollo científico y tecnológico haya sido lento y dependiente del extranjero. [...].

La Universidad no debe actuar en la sociedad sólo a través de sus egresados, sino que tiene que hacerlo, además, como institución, a través de la extensión universitaria (BOENINGER, 1970, p. 3).

A su vez, estos puntos de crítica presentaron una visión democrática y otra oligárquica dentro de la misma universidad. Tal situación es lo que produjo el avance del movimiento democrático en la universidad. Representación de aquello es la posición del ex Rector Juan Gómez Millas, recordado por su "modernización institucional", en sus funciones de Ministro de Educación para el Gobierno de Eduardo Frei:

Resoluciones tales como las que se refieren a la participación de un determinado porcentaje de estudiantes en la generación de las autoridades de la Universidad Técnica del Estado no sólo afectaría a ésta, sino que, a todas las demás Universidades públicas y privadas del país, al progreso de las ciencias y las técnicas y repercutiría en forma profunda y amplia en todo el desarrollo nacional. Por tanto, esto no puede ser resuelto sino con un mismo criterio para todo el sistema de Educación Superior.

Existe la tendencia en los grupos más débiles de las Universidades 
chilenas a dejarse arrastrar por el camino fácil de las concesiones inmaduras a la presión de grupos transitorios, que no representan el consenso general de las comunidades académicas, sino que son más bien reflejo de posiciones contingentes. La verdadera y alta misión de las Universidades es orientar a toda la comunidad nacional con métodos efectivos y de alta productividad intelectual, lo cual depende principalmente de la calidad de las personas que allí trabajan (CASALI, 2015, p. 47).

De esta forma, entendido el desarrollo y el progreso del país como una tarea de personas selectas es que se levanta una oposición democratizadora, en la Universidad de Chile, que reinterpreta la función formativa de la universidad pública desde el concepto y las funciones institucionales específicas de la extensión.

La investigación enriquece a la docencia y ésta sirve de comunicación a los alumnos, a la vez que permite una formación más sólida, abierta a las nuevas concepciones. La extensión cultural permite la difusión del conocimiento a la comunidad nacional y la recepción de los valores de ésta, para asimilarlos como parte integrante de la vida académica. De allí que se supere definitivamente la antigua separación de las funciones universitarias, por estimar que representa un obstáculo a su pleno desarrollo (BOENINGER, 1971, p. 9).

Otra de las Universidades sujetas a procesos de democratización es la Universidad de Concepción. Esta universidad de origen privado representa a una élite regional de marcada trayectoria nacional en diversos procesos políticos y culturales. Tal situación, posiciona a la Universidad de Concepción dentro del concierto de casas de estudios significativas del país. Es así como, al igual que la Pontificia Universidad Católica, la Universidad de Chile y la Universidad Técnica del Estado, la Universidad de Concepción venía desarrollando, desde la década del 50, una serie de procesos de complejidad institucional, pero sin una amplia participación estamental (PÉREZ \& VALLEJOS, 2013, p. 89-92).

En esta dirección, y tras la promoción de la modificación de estatutos y posterior renuncia del rector David Stitchkin, se celebra la primera elección de 
rector en el mes de diciembre de 1968. Tal elección trajo la victoria de Edgardo Enríquez.

El eje central de este proceso democratizador estuvo contenido en la promoción de una administración que dotaría de resguardos y procedimientos claros a una comunidad que vivía sometida a los designios de una gestión corporativa privada. En palabras del Edgardo Enríquez:

\begin{abstract}
Así me parece a mí también, pero ocurre que no siempre las cosas fueron llevadas de esta manera justa, regular y no arbitraria en la Universidad de Concepción. Antes de nuestra administración, hubo profesores, directores de Institutos, jefes de oficinas y altos funcionarios que fueron despedidos hasta con violencia, sin ser escuchados, sin haber tenido la oportunidad de defenderse, sin haber encontrado ante quien apelar. Hubo tres ilustres profesores, uno de ellos director de Instituto, que, por haber apelado a los tribunales de Justicia a propósito de sueldos, fueron destituidos. Hasta hubo un presidente de la asociación del personal que, sorpresivamente de la noche a la mañana, se encontró en la calle. Poco antes, un Instituto entero presento la renuncia y se fue. iesa era la Universidad que algunos añoran y que otros sueñan con recuperar!12
\end{abstract}

En definitiva, el movimiento democratizador, gatillado entre 1967 y 1968, luchó de manera permanente por redefinir de forma democrática cuestiones como la ciencia, la religión, la formación profesional, la investigación, el Estado y su vinculación con el medio, la participación deliberativa de los estamentos y la conformación de una administración cobijada por un derecho laboral democrático. Cada uno de estos procesos presentaron respuestas oligárquicas y formas absolutamente reactivas a cualquier tipo de cambio, por lo que el proceso político del país, como telón de fondo, jugó un papel central en los avances y retrocesos del movimiento democrático en Educación Superior y explican, en buena medida, la reacción que tuvo la posterior dictadura civil-militar por intervenir a las universidades.

\footnotetext{
${ }^{12}$ Homenaje de la Universidad de Concepción al ex rector Edgardo Enríquez y al ex vicerrector Galo Gómez, forjadores de la reforma universitaria. Obtenido (17/04/2017): http://www.memoriachilena.cl/archivos2/pdfs/MCo027774.pdf, p. 33.
} 


\section{REFLEXIONES FINALES}

Las transformaciones de la educación superior chilena hasta el golpe de estado civil-militar deben ser vistas como una trayectoria de democratización de la Educación Superior iniciadas desde 1920, sustentada por diversos procesos de cambios institucionales enmarcados en discusiones de facultades, departamentos, institutos o en el nivel general de las casas de estudios hasta su continuidad en el proceso denominado reforma universitaria en los años 1967 a 1968.

De esta forma, el movimiento democratizador de la educación superior pujó por dar contenido disciplinar y social a las diversas especialidades del conocimiento junto a un compromiso social con el país en él se suceden una serie de cambios institucionales (o reformas) que van paulatinamente diseñando, configurando universidades, robusteciendo su quehacer científico, ampliando su cobertura y regulación pública a través del Estado y promoviendo la educación como valor universal de múltiples beneficios para toda la humanidad.

Este proceso general (ciencia, control público y educación universal) tuvo su mayor expresión en la disputa que cada comunidad educativa vivió en lo que se ha denominado la reforma universitaria (1967-68), debido principalmente al quiebre, transitorio, de las jerarquías internas de las instituciones de educación superior que promovían y sustentaban nociones religiosas o instructivas de la ciencia, promoción de la regulación privada por sobre la pública y la educación como un valor exclusivo de algunos grupos sociales.

Este hito que significó la reforma universitaria de 1967 también debe ser visto como un complejo proceso en que cada institución de educación superior convocó voluntades en pos de cambios democratizadores significados para cada una de ellas.

En este sentido, la democratización de la Pontificia Universidad Católica 
tuvo como eje central la redefinición de la misión católica en un sincretismo con el conocimiento científico y teológico.

Para la Universidad Técnica del Estado se buscó rescatar lo profesional de una formación poco nutritiva y volver a "llenarla" de los principales elementos de avanzada científica y técnica de la época con un sentido de servicio social amplio.

Para la Universidad de Chile el desarrollo y el progreso del país fue visto como una tarea de personas selectas y por eso se levanta una oposición democratizadora, en la propia universidad, que reinterpreta la función formativa de la universidad pública desde el concepto y las funciones institucionales específicas de la extensión universitaria y dota de contenido social la tercera misión para modificar la formación exclusiva de grupos sociales privilegiados.

Y finalmente, la Universidad de Concepción su proceso democratizador estuvo situado en la promoción de una nueva administración que dotaría de resguardos y procedimientos claros a una comunidad que vivía sometida a los designios de una gestión corporativa privada.

\section{REFERENCIAS}

ALLARD, Raúl. La reforma de la Universidad Católica de Valparaíso. In:

CIFUENTES, Luis. La reforma universitaria en Chile: 1967-1973. Santiago de Chile: Editorial Universidad de Santiago, 1997.

ANGELL, Alan. Partidos políticos y movimiento obrero en Chile. De los orígenes hasta el triunfo de la Unidad Popular. Santiago de Chile: Ediciones Era, 1972.

AUSTIN, Robert. Intelectuales y educación superior en Chile. De la independencia a la democracia transicional (1810-2001). Santiago de Chile: Cesoc, 2004.

BARRERA, Manuel. Trayectoria del movimiento de reforma universitaria en 
chile. Journal of inter-american studies, v. 10, n. 4, p. 617-636, oct. 1968.

BASTÍAS, Ignacio. Movimiento anarquista y estudiantes libertarios de los años 20. Hombre y sociedad, n. 21, p. 20-22, 2007.

BIAGINI, Hugo E. Redes estudiantiles en el cono sur (1900-1925). Revista Universum, Universidad de Talca, n. 17, p. 279-296, 2002.

BRUNNER, José Joaquín. Informe sobre el desarrollo y el estado actual del sistema universitario en Chile. Documento de trabajo, Santiago de Chile: Flacso-Chile, 1984.

CÁRDENAS TOMAZIC, Ana; NAVARRO OYARZÚN, Camilo. El movimiento estudiantil en Chile. Redefiniendo límites, acortando distancias. Santiago de Chile: RIL Editores, 2013.

CASALI, Aldo. Reforma, modernización y conflicto político en la Universidad de Chile, 1963-1973. Historia 396, n. 21, p. 37-71, 2015.

CIFUENTES, Luis. El movimiento estudiantil chileno y la reforma unievrsitaria: 1967-1973. In: AUSTIN HENRY, Robert. Intelectuales y Educación

Superior en Chile: de la independencia a la democracia transicional. 18102001, Santiago de Chile: Cesoc, 2004. p.133-151.

CORREA, Sofía. El corporativismo como expresión política del socialcristianismo. Teología y vida, v. 49, n. 3, p. 467-481, 2008.

CPU. Movimientos universitarios y generación de elites dirigentes. Estudios de casos. Santiago de Chile: Corporación de Promoción Universitaria (CPU), 1973.

CRISTI, Renato; RUIZ, Carlos. El pensamiento conservador en Chile. Seis ensayos. Santiago de Chile: Editorial Universitaria, 1992.

DOMÈNECH, Antoni. De la ética a la política. De la razión erótica a la razón inerte. Barcelona: Crítica, 1989.

DOMÈNECH, Antoni. El eclipse de la fraternidad. Una revisión repúblicana de la tradición socialista. Barcelona: Crítica, 2004.

GARCÉS, Mario. Los movimientos sociales populares durante el siglo XX: balance y perspectivas. Revista Política, n. 43, p. 13-33, 2004.

GARRETÓN, Manuel Antonio. Universidad y política en los procesos de transformación en Chile, 1967-1973. Pensamiento universitario, n. 14, p. 7190, oct. 2011. 
GARRETÓN, Manuel; MARTINEZ, Javier. El movimiento estudiantil: concepto e historia. Tomo IV. Santiago de Chile: Ediciones Sur, 1985.

GAZMURI, Cristian. Documento de trabajo n. 3: Notas sobre las élites chilena, 1930-1999. Santiago de Chile: Insituto de Historia. Pontificia Universidad Católica de Chile, 2001.

GONZÁLEZ VERA, José Santos. Estudiantes del año veinte. Babel, n. 28, jul./ago. 1945.

GRAMSCI, Antonio. La formación de los intelectuales. México D.F: Editorial Grijalbo, 1967.

HUNEEUS, Carlos. La reforma universitaria, veinte años después. Santiago de Chile: Corporación de Promoción Universitaria, 1988.

KIRBERG, Enrique. Los nuevos profesionales. Educación universitaria de trabajadores. Chile. UTE, 1968-1973. México D.F: Universidad de Guadalajara, 1981.

MORRIS, James O. Las elites, los intelectuales y el consenso. Estudio de la cuestión social y del sistema de relaciones industriales en Chile. Santiago de Chile: Editorial del Pacifico, 1966.

MOYANO, Cristina. MAPU o La seducción del poder y la juventud. Los años fundacionales del partido - mito de nuestra transición (1969 - 1973). Santiago de Chile: Universidad Alberto Hurtado, 2009.

MUÑOZ TAMAYO, Victor. Chile es bandera y juventud. Efebolatría y gremialismo durante la primera etapa de la dictadura de Pinochet (1973-1990). Historia Crítica, n. 54, p. 195-219, 2014.

RIVERA TOBAR, Francisco. Laicos, católicos y técnicos: análisis comparativo de las experiencias de reforma universitaria en las universidades de Chile, católica y técnica del estado. Chile 1960-1973. Santiago de Chile: Consejo nacional de educación, 2013.

ROJAS, Gonzalos. Textos fundamentales sobre la universidad. Santiago de Chile: Tercer Edición, 2012.

SCHOFER, Evan; MEYER, Jhon W. The worldwide expansion of higher education in the twentieth century. American sociological review, v. 70, p. 898-920, dec. 2005.

SCHWEITZER, Daniel. La justicia y los procesos de los subversivos. Claridad, 
v. 1, n. 23, julio 1921.

SILVA, Beatriz. Democracia y conflicto. La democracia como movimiento histórico. Revista latinoamericana Polis, v. 16, n. 46, p. 77-98, 2017.

SUBERCASEUX, Bernardo. Editoriales y círculos intelectuales en Chile 19301950. Revista chilena de literatura, n. 72, p. 221-233, abril 2008.

VALDIVIA DE ZARATE, Verónica. Nacionales y gremialistas. El "parto" de la nueva derecha política chilena. 1964-1973. Santiago de Chile: LOM, 2008.

VALENZUELA VAN TREEK, Esteban. El Mapu y el rol transformador de las élites ilumnistas: revolución, pragmatismo y disidencia. Revista de Ciencia Política, v.31, n. 2, p. 187-206, 2011.

VALENZUELA VAN TREEK, Esteban. Cristianismo, revolución y renovación en Chile: el movimiento de acción popular unitaria (Mapu) 19691989. 2011. Tesis (Doctorado en Historia) - Universitat de Valencia, Valencia, 2011.

MAURICIO ESTEBAN RIFO es Doctor en Educación en la Universidad Autónoma de Barcelona y Magíster en Educación, Mención Gestión Cultural de la Escuela en la Universidad de Arte y Ciencias Sociales. Profesor de Historia y Geografía de la Universidad de Concepción. Cuenta también con un Postgrado en Filosofía Política y Capitalismo Contemporáneo en la Universidad de Barcelona. Ha publicado en diversas revistas sobre Historia de la Educación y movimientos estudiantiles en Chile.

E-mail: mauricioesrifo@gmail.com

(ib) http://orcid.org/0000-0002-5627-1884

Recebido em: 07 de dezembro de 2017

Aprovado em: 11 de junho de 2019 